\title{
Psycho-cognitive syndrome, blindness and tetraplegia after severe traumatic brain injury in polytraumatic context (road acc.) with favorable recovery of cognitive and motor deficits
}

\author{
Andreea Dumitrascu ${ }^{1}$, Iulia-Maria Nohai ${ }^{2}$, Simona Isabelle Stoica ${ }^{1}$, Carmen Cihpăruș ${ }^{1}$; \\ Magdalena Lapadat ${ }^{1}$, Ioana Andone', Gelu Onose ${ }^{1,2}$
}

Corresponding author: Andreea Dumitrascu, E-mail address: dumitrascu.andreea0417@gmail.com

${ }^{1}$ The Teaching Emergency Hospital “Bagdasar-Arseni” (TEHBA), Bucharest, Romania ${ }^{2}$ The University of Medicine and Pharmacy "Carol Davila” (UMPCD), Bucharest, Romania

\begin{abstract}
Introduction

The impact of accidents is important both for younger and older people. We live in a multisensory environment and the interaction between our genes and the environment shapes our brains. Cortical blindness as a result of head trauma (to the brain's occipital cortex) is a rare phenomenon and can be a total or partial loss of vision in a normal-appearing eye. How patients will adjust to the loss of vision and its consequences might be a challenge let along if they have mobility imparement (tetraplegia) as well. Adaptation and reintegration of patients into society after motor recovery in the context of visual sensory deficit. TBI survivors themselves and their families are likely more interested in quality-of-life outcomes, such as reintegration into the community, successful return to work or school, and functional capacity in everyday life. Cognitive and behavioural changes, difficulties maintaining personal relationships and coping with school and work are reported by survivors as more disabling than any residual physical deficits.

As with all rehabilitation, the goal is to help the person achieve the maximum degree of return to their previous level of functioning.

Case presentation

Having the patient and TEHBA Bioethics Committee aproval, we will present the evolution of a case with postraumatic spastic tetraplegia post severe traumatic brain injury, blindness post traumatic bilateral occipital lesions and psycho-cognitive syndrome. Clinical and paraclinical aspects will be discussed (patient history and clinical examination, results of imaging and laboratory tests, the neuromioartrokinetic exam, specific rating scales, both medical and kinetotherapeutic treatments). We will address the case in terms of particularities and treatment approach (neurorehabilation of a motor deficit in the context of a major sensory deficiency) and evolution during hospitalization.

\section{Conclusions}

Trauma has been known to result in cortical blindness but the exact pathophysiology remains unknown and remains a matter of continued debate. Cortical blindness may occur after trauma, however, most cases regardless of etiology, are reversible and have no long term sequelae. While TBI can cause long-term physical disability, it is the complex neurobehavioural sequelae that produce the greatest disruption to quality of life. As with all rehabilitation, the goal is to help the person achieve the maximum degree of return to their previous level of functioning. In the setting of polytrauma, a careful ophthalmologic and neurologic examination of the trauma patient, together with a high index of suspicion, is necessary for the diagnosis of this condition. Heightened awareness of the causes should be followed with appropriate imaging and management.

Key words: Traumatic brain injuries, spinal cord injury Polytraumatism

\section{Introduction}

Traumatic brain injury (TBI) refers to blunt, penetrating, or acceleration/deceleration forcederived cranio-cerebral injury, which causes symptoms such as decline in level of awareness or consciousness, memory loss or forgetfulness, other neurological or neuropsychological abnormalities, and even death. According to the World Health Organization, TBI will be a major health problem and the main reason for disability in 2020 . Primary and secondary TBIs cause temporary and/or permanent dysfunction in the brain, which limits a patient's activities, affects participation in society, and lowers quality of life. This can lead to depression and other chronic diseases in TBI patients . ${ }^{[3]}$
TBI can cause persistent, sometimes life-long, consequences. While mortality after TBI has been reduced, a high proportion of severe TBI survivors require prolonged rehabilitation and may suffer longterm physical, cognitive, and psychological disorders. Additionally, chronic consequences have been identified not only after severe TBI but also in a proportion of cases previously classified as moderate or mild. This burden affects the daily life of survivors and their families; it also has relevant social and economic costs. ${ }^{[4]}$ We will include a list of symptoms for TBI: Headaches, Dizziness, Confusion, Convulsions, Loss of coordination, Slurred speech, Poor concentration, Memory problems, Personality 
changes and Visuall loss which is the subject of the article.

Cortical blindness as a result of head trauma (to the brain's occipital cortex) is a rare phenomenon and can be a total or partial loss of vision in a normalappearing eye. Visual rehabilitative metodes: visual aids aim to expand visual field; eye movement training improve explorative saccades; visual field restitution aims to improve visual processing within the damaged field itself. ${ }^{[1]}$ Rehabilitation helps TBI patients in their healthcare complex to exceed their marked fragility so, that, after a few weeks or months (in some cases, even 1-2 years) they can reach biological stabilization, eventually adapting to new capacities, (dis)functional - permissive to continue to support / benefit from physical / kinetic programs / effective recuperators. ${ }^{[2]}$

Rehab can take many forms depending on the pacients needs, and includes physical, occupational, and speech therapy, as well as psychiatric care and social support. ${ }^{[5]}$

The approach for the patient with post-traumatic visual loss is complicated by a wide range of potential ocular and brain injuries with varying pathophysiology. Classic causes of visual loss after head trauma can be: direct injuries of the eye and orbit, traumatic optic neuropathies, carotid cavernous fistulas, and damage to the intracranial visual pathways [6] Visual loss can interfere with rehabilitation, and many trauma patients are unaware of or unable to complain of visual loss, it is important that formal evaluation of visual function, including visual field testing, be obtained in head trauma patients during rehabilitation. Indeed, documentation of visual function is essential for proper evaluation of the ability to return to school or work, and to drive a vehicle. ${ }^{[6]}$

\section{CASE PRESENTATION}

We present a case of 52 year-old pacient, who suffers on september 2017 a polytrauma with sever TBI and cortical blindeness after a road accident (pedestrian). Neurosurgical interventions are performed: FTP decompressive craniectomy. Orthopedic intervention occurs for the left humerus fracture. She is hospitalized in the SCUBA Neuromuscular Recovery Section for specialized treatment on December 13, 2017.

The reasons for admission on Neuromuscular Recovery Section: Predominantly crural spastic tetraplegia after a severe TBI following a road accident (sept 2017). Neurological Bladder. Decompressor craniectomy. Left humerus fracture.

Clinical exam \& NMK exam: General mediocre status, post traumatic condition, GCS $=11$, afebrile, mixed aphasia, temporo- spatial disorientation, underweight, pale teguments, sacrum decubital ulcer. Upper limbs normal, lower limbs significant atrophy of the thighs and legs muscles, weak intermedialdistal control, bilateral. Static vertebral syndromekhyphosis; Dinamic: difficult to clinically appreciate. Dural syndrome-negative. Neuroradicular syndromefunctional tetraparesis syndrome. Increased deep tendon reflex. Babinski sign is present.

Respiratory \& Cardiovascular System: bilaterally vesicular murmur present, without bronchial rallies, SaO2: 93\%, rhythmic heart rhythm, no signs of heart failure, TA: 110 / 75mmHg, bilateral thrombophlebitis on lower limbs.

Digestive system: Slow abdomen, mobile with breathing, intestinal transit was present, slow, lower limb of the liver at the RC level, non-palpable spleen. Urogenital System: Urinary retention - mounts Foley catheter

The blood tests showed anemic syndrome (HTC = 28.86\% and $\mathrm{Hb}=9.11 \mathrm{~g} / \mathrm{dL}$ ) and Biological inflammatory syndrome (VSH $=90 \mathrm{~mm} / \mathrm{h}$, fibrinogen $=526 \mathrm{mg} / \mathrm{dl}$ ), hypoalbuminemia (albumin $=2.2 \mathrm{~g} / \mathrm{dl}$ ), hypertriglyceridemia $=173 \mathrm{mg} / \mathrm{dl}$ ).

The CT scan shows us a postsurgical cerebral state with frontal-temporo-parietal decompressive craniotomy, at which is highlighted the densification of the dura mater and minimal areas with spontaneous hipodensitys, fatty axonal maximal 6 / 9mm diameter. Dilations and tractions on the anterior and posterior horns of the lateral ventricles. Capsulolenticular and right thalamus straps. The ventricular system on the median line.

\section{Scales:}

-FIM: 24 motor admission and 27 at discharge; MMSE:11; Asworth: 0 upper limbs and 3 lower limbs; Penn: 2; GOS:3, Rankin: 5; QOL: 80 admission, 96 discharge.

Oftalmology exam: blood vessels of diminished size. Visual evoked potential: 0 electrical signal recorded.

Evolution of the patient: Slowly favorable evolution. The patient underwent medical treatment with: antithrombotics for prophylaxis of TEP, antispasmodic, antibiotic, neurotrophic. An appropriate kinetotherapeutic program: Passive and active gymnastics, gradually mobilized with the bed, 
at the bedside and rolling chair, coordination and balance exercises, verticalized with bilateral support, practiced electric bicycle exercises, practiced walking at parallel bars by shifting weight from side to side and from front to back with the support of the physical therapist then progressed to walking with mechanical aids, such as a walker, crutches. She followed a speech therapy program. Cognitive rehabilitation was a very slow process, the pacient had verbal, visual, and tactile cues just so she can learn and remember how to do the tasks. The visual deficit is maintained.

\section{DISCUSSIONS:}

After the patient became aware and understood the situation, she had much more progress in rehabilitation. The discussion starts as what can we do for the pacient that went blind after a TBI? The goal is to help people function as well as possible, become independent, and regain their selfconfidence. The main goal of rehabilitation is to help the pacient to regain mobility without support of any accompanying person, at home, in public and private buildings, and in open spaces. Mobile and wireless technologies such as those used to locate persons or objects can be used as navigation systems so that pacients can navigate space independently, safely and efficiently in a combined product of motor, sensory and cognitive skills which will open new opportunities to improve the speed, easiness, and safety of the visually impaired persons mobility. ${ }^{[7]}$

We need to understand that the pacient needs to be socially integrated after the hospitalization and needs to be more and more independent. Research in visual impairment and blindness is aimed at developing and assessing new methods for the rehabilitation of visually impaired individuals through assistive technologies, training, and rehabilitation services and education ${ }^{[8]}$

\section{CONCLUSIONS:}

More virtual reality applications have been developed in the past years which could minimize the effects of a disability, improve quality of life, enhance social participation, and improve life skills, mobility and cognitive abilities, while providing a motivating and interesting experience. ${ }^{[8]}$

Although patients can largely recover through kinetotherapy and physiotherapy, there are still no well-defined methods on the visual rehabilitation side. We hope that ongoing studies will come to a favorable conclusion for these patients.

\section{Bibliography}

1. Ajina S1, Kennard C. - Rehabilitation of damage to the visual brain. Rev Neurol (Paris). 2012

2. Onose G. - Recuperare, Medicină Fizică si Balneoclimatologie - noțiuni de bază și actualități Vol. I. Ed. Medicală, București, 2007

3. Baoqi Dang,1,2 Wenli Chen, Weichun He,2 and Gang Chen1-Rehabilitation Treatment and Progress of Traumatic Brain Injury Dysfunction

4. Nino Stocchetti, Elisa R. Zanier - Chronic impact of traumatic brain injury on outcome and quality of life: a narrative review

5. JOHNS HOPKINS MEDICINE https://www.hopkinsmedicine.org/healthlibrary/cond itions/nervous_system_disorders/rehabilitation_after _traumatic_brain_injury_135,2

6. Atkins EJ, Newman NJ, Biousse V. Post-Traumatic Visual Loss. Reviews in neurological diseases. 2008;5(2):73-81.

7. E. D'Atri, C. M. Medaglia, A. Serbanati, U. B. Ceipidor, E. Panizzi and A. D'Atri, "A system to aid blind people in the mobility: A usability test and its results," Second International Conference on Systems (ICONS'07), Martinique, 2007, pp. 35-35.

8. Neveen I. Ghali1, Omar Soluiman2, Nashwa ElBendary3,Tamer M. Nassef4, Sara A. Ahmed1, Yomna M. Elbarawy1,And Aboul Ella Hassanien1 Virtual Reality Technology for Blind and Visual Impaired People: Reviews and Recent Advances

9. J. M. Mazaux \& E. Richer (1998) Rehabilitation after traumatic brain injury in adults, Disability and Rehabilitation, 20:12, 435-447.

10. Munteanu C. - Cell biology considerations in Spinal Cord Injury - Review Balneo Research Journal. 2017;8(3):136-151 DOI 10.12680/balneo.2017.149 